\title{
Analytic continuation of finite density QCD with heavy quarks in the strong coupling region
}

\section{Shinji Ejiri}

Graduate School of Science and Technology, Niigata University, Niigata 950-2181, Japan

E-mail: ejiri@muse.sc.niigata-u.ac.jp

\section{Hiroshi Yoneyama*}

Department of Physics, Saga University, 840-8502 Saga, Japan

E-mail: yoneyama@cc.saga-u.ac.jp

Complex nature of finite density QCD with heavy quarks in the strong coupling region is studied. For this purpose, we consider the effective potential as a function of Polyakov line, and study thermodynamic singularities and associated Stokes boundaries in the complex chemical potential plane. We also perform an explicit analytic continuation of the first order transition and crossover lines in the complex $\mu$ plane.

The 33rd International Symposium on Lattice Field Theory

14 -18 July 2015

Kobe International Conference Center, Kobe, Japan*

\footnotetext{
${ }^{*}$ Speaker.
} 


\section{Introduction}

In this talk, we discuss the phase structure in the quark complex chemical potential $(\mu)$ plane of the Polyakov line model, which is an effective theory of finite density QCD with heavy quarks in the strong coupling region. The purpose of studying in the complex $\mu$ plane is twofold. One is that the Lee-Yang zeros [1, 2, 3], the edge singularities and the Stokes boundaries are deeply associated with the phase structures, and may provide useful information of the QCD critical point [4, 5, 6]. The other concerns the validity of the imaginary chemical potential method used in the Monte Carlo simulations $[7,8,9,10,11,12,13,14,15,16]$, which relies on the analytic continuation in the complex $\mu$ plane.

In the space of parameters associated with the hopping parameter, the gauge coupling and $\mu$, this model reveals an interesting structure involving first order phase transition lines, crossovers and critical endpoints. To discuss their complex nature, we calculate the effective potential as a function of the Polyakov line, adopting a variational method based on the Legendre transformation in the mean field framework. By extending $\mu$ to the complex plane, we investigate thermodynamic singularities, the edge singularities and the associated Stokes boundaries. Our study may hopefully provide a useful view of the expected QCD critical point.

In the following section, the effective potential is calculated. In Sect. 3, we study the model in the complex $\mu$ plane. Critical endpoint as a singularity, the edge singularity, crossover and Stokes boundaries are discussed. The first order phase transition line is analytically continued to the complex plane. Conclusions are given in Sect. 4.

\section{Effective potential}

The Polyakov line model, which is an effective theory of finite temperature and finite density QCD in the strong coupling region at the leading order in the hopping parameter expansion, is defined by

$$
\begin{aligned}
& Z=\int[d U] e^{-S} \\
& S=-\beta_{p} \sum_{x, x^{\prime}} \sum_{i=1}^{d} \operatorname{Tr} U_{x} \operatorname{Tr} U_{x^{\prime}}^{\dagger}+\text { c.c. }-\kappa \sum_{x}\left(e^{\mu} \operatorname{Tr} U_{x}+e^{-\mu} \operatorname{Tr} U_{x}^{\dagger}\right),
\end{aligned}
$$

where $\beta_{p}$ and $\kappa$ are two parameters defining the model, and the $\mu$ is chemical potential. The space dimensionality $d$ is fixed to 3 throughout the calculations. In this report we use the mean field theory based on the variational method in terms of the Legendre transformation by introducing two variational parameters $K$ and $\hat{K}$ to the partition function ${ }^{1}$;

$$
\begin{aligned}
& Z=\int[d U] \exp \left[\beta_{p} \sum_{x, x^{\prime}} \sum_{i=1}^{d} \operatorname{Tr} U_{x} \operatorname{Tr} U_{x^{\prime}}^{\dagger}+\text { c.c. }-K \sum_{x} \operatorname{Tr} U_{x}-\hat{K} \sum \operatorname{Tr} U_{x}^{\dagger}\right] \\
& \times \exp \left[(h+K) \operatorname{Tr} U_{x}+(\hat{h}+\hat{K}) \operatorname{Tr} U_{x}^{\dagger}\right],
\end{aligned}
$$

\footnotetext{
${ }^{1}$ Greensite and Splittorff 17 has investigated the model using the mean field method in the different framework from ours and found that its phase diagram agrees fairly well with that of Monte Carlo simulations [18].
} 
where $h=\kappa e^{\mu}$ and $\hat{h}=\kappa e^{-\mu}$. Defining a partition function characterize by $K$ and $\hat{K}$ with the site-independent measure on a $N_{s}$-site lattice

$$
Z_{K, \hat{K}} \equiv\left(z_{K, \hat{K}}\right)^{N_{s}}, \quad z_{K, \hat{K}}=\int[d U] e^{(h+K) \operatorname{Tr} U+(\hat{h}+\hat{K}) \operatorname{Tr} U^{\dagger}},
$$

$Z$ is interpreted as an expectation value

$$
Z=\left\langle\exp \left[\beta_{p} \sum_{x, x^{\prime}} \sum_{i=1}^{d} \operatorname{Tr} U_{x} \operatorname{Tr} U_{x^{\prime}}^{\dagger}+\text { c.c. }-K \sum_{x} \operatorname{Tr} U_{x}-\hat{K} \sum \operatorname{Tr} U_{x}^{\dagger}\right]\right\rangle_{K, \hat{K}} Z_{K, \hat{K}},
$$

where the expectation value $\langle\cdot\rangle_{K, \hat{K}}$ specified by $K$ and $\hat{K}$ is taken with respect to Eq. (2.4). Application of the Jensen's inequality $\left\langle e^{A}\right\rangle \geq e^{\langle A\rangle}$ to Eq. (2.5) leads to an inequality concerning the free energy density $f=-\left(1 / N_{s}\right) \log Z$

$$
f \leq f_{K, \hat{K}}
$$

where $f_{K, \hat{K}}$ is an approximate free energy density

$$
f_{K, \hat{K}} \equiv-\log z_{K, \hat{K}}-\beta_{p} d\left(\langle\operatorname{Tr} U\rangle_{K, \hat{K}}\left\langle\operatorname{Tr} U^{\dagger}\right\rangle_{K, \hat{K}}+\text { c.c. }\right)+K\langle\operatorname{Tr} U\rangle_{K, \hat{K}}+\hat{K}\left\langle\operatorname{Tr} U^{\dagger}\right\rangle_{K, \hat{K}} .
$$

The parameters $K$ and $\hat{K}$ are fixed so as to minimize $f_{K, \hat{K}}$ as the best approximation, and consequently, we have

$$
f_{\bar{K}, \bar{K}}=-\log z_{\bar{K}, \bar{K}}+\frac{1}{2 \beta_{p} d} \bar{K} \overline{\hat{K}}
$$

where

$$
\bar{K}=2 \beta_{p} d\left\langle\operatorname{Tr} U^{\dagger}\right\rangle_{\bar{K}, \bar{K}}, \quad \overline{\hat{K}}=2 \beta_{p} d\langle\operatorname{Tr} U\rangle_{\bar{K}, \overline{\hat{K}}}
$$

In order to have the effective potential $\Omega(M)$ as a function of expectation value of $M=\langle\operatorname{Tr} U\rangle$ through the Legendre transform of $f_{\bar{K}, \bar{K}}$, we introduce an "external field" $h_{e}$ conjugate to $M$ so that

$$
\frac{\partial f_{\bar{K}, \overline{\hat{K}}}}{\partial h_{e}}=-M
$$

Inverting Eq. (2.10) to obtain $h_{e}(M), f_{\bar{K}, \overline{\hat{K}}}$ is Legendre-tranformed to $\Omega(M)$

$$
\Omega(M)=f_{\bar{K}, 2 M \beta_{p} d}\left(h_{e}(M)\right)+h_{e}(M) M,
$$

where

$$
\frac{\partial \Omega(M)}{\partial M}=h_{e}
$$

Fig. 1 indicates, as a function of $\beta_{p}$, the expectation values of $\operatorname{Tr} U$ and $\operatorname{Tr} U^{\dagger}$, the former is identified as a global minimum of $\Omega(M)$ satisfying $h_{e}=0$ in Eq. (2.12), and the latter from the corresponding $\overline{\hat{K}} /\left(2 \beta_{p} d\right)$. It is seen that a first order phase transition occurs at $\beta_{p}=0.12579$. In Fig. 2. the first order phase transition lines and the critical endpoints are shown for $\kappa=0.02$ and 0.05 in $\mu-\beta_{p}$ plane. As $\kappa$ goes up, the first order phase transition line shrinks and disappears at $\left(\mu, \beta_{p}\right) \approx(0,0.12068)$ for $\kappa_{c} \approx 0.05905$. This phase structure is in agreement with that in [17]. 


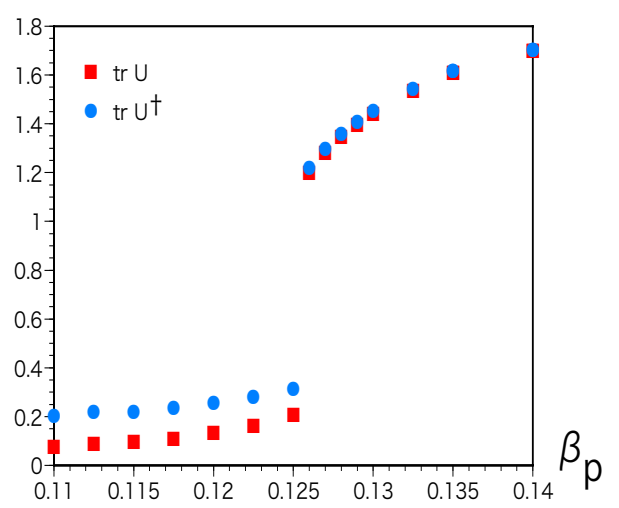

Figure 1: Expectation values of $\operatorname{Tr} U$ and $\operatorname{Tr} U^{\dagger}$ as a function of $\beta_{p} . \kappa=0.02$ and $\mu=1.2$.

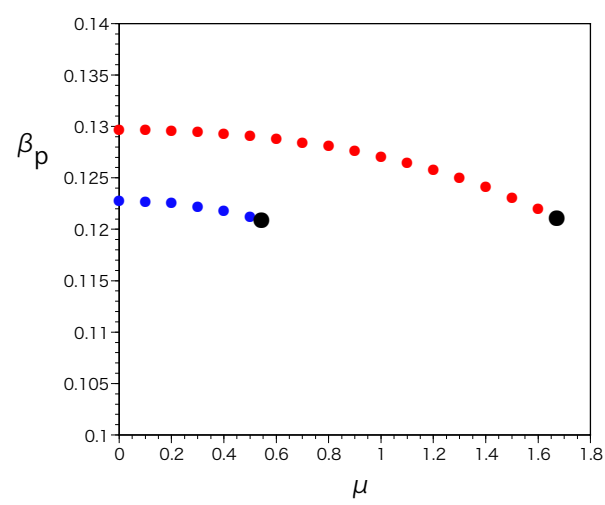

Figure 2: Phase diagram in $\mu-\beta_{p}$ plane. First order phase transition lines are shown for $\kappa=0.02$ (red) and 0.05 (blue). Black filled symbol indicates the critical endpoints.

\section{Complex $\mu$ plane}

\subsection{Singularities}

In this section, we discuss the nature in the complex $\mu$ plane. The singularity occurs when the global minimum of $\Omega(M)$ becomes unstable, i.e., the followings are satisfied

$$
\frac{\partial \Omega(M)}{\partial M}=0, \quad \frac{\partial^{2} \Omega(M)}{\partial M^{2}}=0
$$

which are equivalent to

$$
h_{e}(M)=0, \quad \frac{\partial h_{e}(M)}{\partial M}=0
$$

in our formulation. For the equality on the right hand side in Eq. 3.2. we use an explicit expression for $\partial h_{e} / \partial M$

$$
\frac{\partial h_{e}}{\partial M}=\frac{1}{\left\langle(\operatorname{Tr} U)^{2}\right\rangle_{c}}\left[\left(2 \beta_{p} d\left\langle\operatorname{Tr} U \operatorname{Tr} U^{\dagger}\right\rangle_{c}-1\right)^{2}-\left(2 \beta_{p} d\right)^{2}\left\langle(\operatorname{Tr} U)^{2}\right\rangle_{c}\left\langle\left(\operatorname{Tr} U^{\dagger}\right)^{2}\right\rangle_{c}\right],
$$

where

$$
\langle A B\rangle_{c} \equiv\langle A B\rangle_{\bar{K}, 2 M \beta_{p} d}-\langle A\rangle_{\bar{K}, 2 M \beta_{p} d}\langle B\rangle_{\bar{K}, 2 M \beta_{p} d}
$$


Fig. 3 indicates the locations of the singular points as a solution to Eq. (3.2) in the complex $\mu$ plane for $\kappa=0.02$. The left edge of the plots at $\mu=1.6709 \equiv \mu_{E}\left(\beta_{p}=0.12122\right)$ corresponds to the endpoint of the aforementioned first order phase transition line in Fig. 2 For Re $\mu>\mu_{E}$, a complex conjugate pair appears as the edge singularities corresponding to different value of $\beta_{p}$ $\left(0.117 \leq \beta_{p} \leq 0.12122\right)$. A fit to the upper half part of the singular points in Fig. 2 give $\operatorname{Im} \mu \propto$ $\operatorname{Re}\left(\mu-\mu_{E}\right)^{3 / 2}$.

Moving away of the singularities from the real axis for $\operatorname{Re} \mu>\mu_{E}$ causes a crossover phenomenon on the real $\mu$ axis. In agreement with what was shown in [6], it turns out that the location of the crossover, which is measured as a peak of the susceptibility $\chi$ associated with the Polyakov loop

$$
\chi \equiv 1 /\left.\left(\frac{\partial^{2} \Omega(M)}{\partial M^{2}}\right)\right|_{\bar{M}}
$$

agrees with the real part of the location of the singularity in the complex $\mu$ plane.

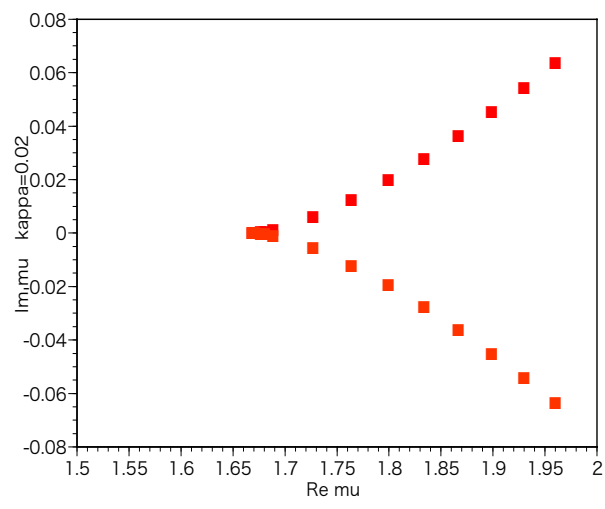

Figure 3: Locations of the singular points for $\kappa=0.02$ in the complex $\mu$ plane. The critical point is located at the left edge of the plots $\left(\mu=1.6709 \equiv \mu_{E}\right)$.

\subsection{Stokes boundaries}

For each singular point, an associated cut, the Stokes boundary, is connected to it in the complex $\mu$ plane. To locate where it is, we perform the analytic continuation of the global minimum in the complex $\mu$ plane. For this, setting $\mu-\mu_{E}=\rho e^{i \theta}$ and varying $\theta$ from 0 to $\pi$ for every fixed value of $\rho$, we trace the movement of the global minimum as was done in [6]. In the course of variation, a jump of the global minimum occurs at a point where the real part of the potential $\operatorname{Re} \Omega(M)$ satisfies the following

$$
\operatorname{Re} \Omega\left(M_{1}\right)=\operatorname{Re} \Omega\left(M_{2}\right)
$$

where $M_{1}$ and $M_{2}$ are the location of the two different global minima analytically continued from those on the real axis. In Fig. 4, the behaviors of the Stokes boundaries in the neighborhood of $\mu_{E}$ are shown. For $\beta_{p}=0.12123$, the Stokes boundary goes upright $(\varphi=\pi / 2)$ from the origin $(\mu=$ $\left.\mu_{E}\right)$. As $\beta_{p}$ deviates from $\beta_{E}$, the singularity moves away from the real axis, and corresponding Stokes boundary emanates from it with slightly increasing $\varphi$. 


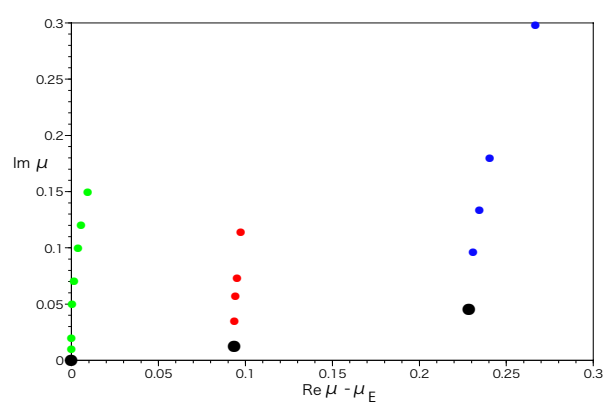

Figure 4: Behaviors of the Stokes boundaries for $\kappa=0.02$. Filled circles (black) are singular points for three values of $\beta_{p}, 0.12123\left(=\beta_{E}\right), 0.12$ and 0.118 , and the Stokes boundary emanates from each of them.

\subsection{Analytic continuation of the first order phase transition line}

In dealing with the sign problem in lattice simulations of finite density QCD, the imaginary chemical potential method relies on the validity of the analytic continuation from the real $\mu$ axis to the imaginary one. In order to explicitly perform the analytic continuation, we study how the first order phase transition line on the real $\mu$ axis persist in the complex plane. For this purpose, we trace the global minimum of $\Omega(M)$ in the complex plane as done in the previous subsection. With the parametrization $\mu=\rho e^{i \theta}$, we vary the value of $\rho$ for a fixed value of $\theta$, and search a location in the complex $\mu$ plane where a jump of the global minimum occurs. Fig. 5indicates the first order phase transition lines in the $\rho-\beta_{p}$ plane for several fixed $\theta$ values in the region of $0 \leq \theta \leq \pi / 2$, where $\kappa$ is fixed to 0.05 . It is found that the first order phase transition line varies smoothly from the real axis $(\theta=0)$ to the imaginary one $(\theta=\pi / 2)$. Monotonically decreasing curve at $\theta=0$ (real $\mu$ axis) gradually turns into increasing one as $\theta$ increases, and for $\theta=\pi / 4$, it becomes almost constant, suggesting a behavior of the first order phase transition surface as $\beta_{p}(\mu) \propto \operatorname{Re} \mu^{2}$.

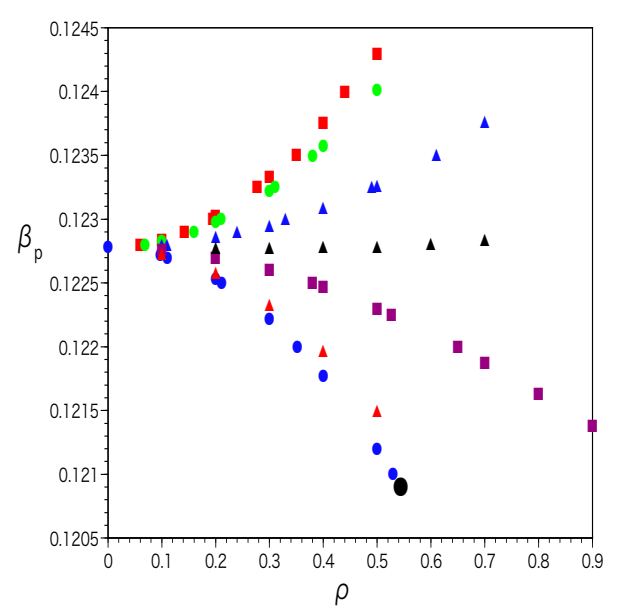

Figure 5: Analytic continuation of the first order phase transition line as a function of $\rho$ in the vicinity of the origin, where $\mu=\rho e^{i \theta} . \kappa=0.05$. Seven values of $\theta$ are chosen; $0, \pi / 10,2 \pi / 10, \pi / 4,3 \pi / 10,4 \pi / 10$ and $\pi / 2$ (from bottom to top). The black filled symbol for $\theta=0$ indicates the critical endpoint on the real $\mu$ axis. 


\section{Remarks}

We investigated the phase structure in the complex chemical potential plane of finite density QCD with heavy quarks in the strong coupling region by adopting the Polyakov line model. We considered the effective potential as a function of Polyakov line, and studied thermodynamic singularities and associated Stokes boundaries in the complex chemical potential plane. By explicitly tracing the global minima, we also performed an analytic continuation of the first order transition lines and found that the transition line is smoothly continued from the real $\mu$ axis to the pure imaginary axis.

Monte Carlo calculations of the effective potential as a function of the Polyakov line of QCD with heavy quarks was done in the literature [19]. Its extension to the complex $\mu$ and the comparison with the results presented here will be presented elsewhere[20].

\section{Acknowledgments}

This work is in part supported by JSPS KAKENHI Grant No. 26400244, No. 26287040.

\section{References}

[1] C. N. Yang and T.D. Lee, Phys. Rev. 87, 404 (1952).

[2] T.D. Lee and C. N. Yang, Phys. Rev. 87, 410 (1952).

[3] C. Itzykson, R.B. Pearson and J.B. Zuber, Nucl. Phys. B 220, 415 (1983).

[4] M. A. Stephanov, Phy. Rev. D 73, 094508 (2006).

[5] S. Ejiri and H. Yoneyama, Proceedings of Science (LAT2009) 173 (2009), arXiv:0911.2257[hep-lat].

[6] S. Ejiri, Y. Shinno and H. Yoneyama, Prog. Theor. Exp. Phys. 2014,083B02 (2014).

[7] M. -P. Lombardo, Nucl. Phys. B (Proc. Suppl.)83, 375 (2000).

[8] P. de Forcrand and O. Philipsen, Nucl. Phys. B 642, 290 (2002).

[9] P. de Forcrand and O. Philipsen, Nucl. Phys. B 673, 170 (2003).

[10] M. D’Elia and M. -P. Lombardo, Phy. Rev. D 67, 014505 (2003).

[11] M. D'Elia and M. -P. Lombardo, Phy. Rev. D 70, 074509 (2004).

[12] P. Cea, L. Cosmai, M. D’Elia and A. Papa, J. High Energy Phys. 02066 (2007).

[13] L.-K. Wu, X.-Q. Luo and H.-S Chen, Phy. Rev. D 76, 034505 (2007).

[14] P. Cea, L. Cosmai, M. D’Elia, C. Manneschi and A. Papa, Phy. Rev. D 80, 034501(2009).

[15] K. Nagata and A. Nakamura, Phy. Rev. D 83, 114507 (2011).

[16] P. Cea, L. Cosmai, M. D’Elia, A. Papa and F. Sanfilippo, Phy. Rev. D 85, 094512 (2012).

[17] J. Greensite and K. Splittorff, Phys. Rev. D 86, 074501 (2012).

[18] Y.D. Mercado and C. Gattringer, Nucl. Phys. B 862, 737 (2012).

[19] H. Saito, S. Ejiri, S. Aoki, K. Kanaya, Y. Nakagawa, H. Ohno, K. Okuno and T. Umeda, Phy. Rev. D 89, 034507 (2014).

[20] S. Ejiri and H. Yoneyama, in preparation. 